\title{
A genome-wide association study identifies FSHR rs2300441 associated with follicle-stimulating hormone levels
}

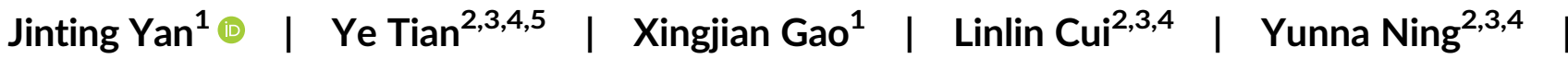 \\ Yongzhi Cao ${ }^{2,3,4}$ | Yan Chen ${ }^{1}$ | Fuduan Peng ${ }^{1}$ | Li You ${ }^{2,3,4}$ | Fan Liü | \\ Han Zhao ${ }^{2,3,4}$
}

\begin{abstract}
${ }^{1}$ CAS Key Laboratory of Genomic and Precision Medicine, Beijing Institute of Genomics, University of Chinese Academy of Sciences, Chinese Academy of Sciences, Beijing, China

${ }^{2}$ Center for Reproductive Medicine, Shandong University, Jinan, China

${ }^{3}$ National Research Center for Assisted Reproductive Technology and Reproductive Genetics, Jinan, China

${ }^{4}$ The Key Laboratory for Reproductive Endocrinology, Shandong University, Ministry of Education, Jinan, China

${ }^{5}$ Department of Gynecology and Obstetrics, Tianjin Medical University General Hospital, Tianjin, China

${ }^{6}$ Department of Genetic Identification, Erasmus MC University Medical Center Rotterdam, Rotterdam, The Netherlands

\section{Correspondence}

Fan Liu, CAS Key Laboratory of Genomic and Precision Medicine, Beijing Institute of Genomics, University of Chinese Academy of Sciences, Chinese Academy of Sciences, Beijing, China. Email: liufan@big.ac.cn
\end{abstract}

Han Zhao, Center for Reproductive Medicine, Shandong University, Jinan, China.

Email: hanzh80@yahoo.com

Funding information

the National Key Research and Development Program of China, Grant/Award Number: 2017YFC1001000; the National Natural Science Foundation of China, Grant/Award Numbers: 31571548, 81622021, 81701410, 31601199; the National Natural Science Foundation of Shandong Province, Grant/ Award Number: JQ201816

Peer Review

The peer review history for this article is available at https://publons.com/publon/10. 1111/cge.13741.

\begin{abstract}
Follicle-stimulating hormone (FSH) and luteinizing hormone (LH) play critical roles in female reproduction, while the underlying genetic basis is poorly understood. Genome-wide association studies (GWASs) of FSH and LH levels were conducted in 2590 Chinese females including 1882 polycystic ovary syndrome (PCOS) cases and 708 controls. GWAS for FSH level identified multiple variants at FSHR showing genome-wide significance with the top variant (rs2300441) located in the intron of FSHR. The A allele of rs2300441 led to a reduced level of FSH in the PCOS group $\left(\beta=-.43, P=6.70 \times 10^{-14}\right)$ as well as in the control group $(\beta=-.35$, $\left.P=6.52 \times 10^{-4}\right)$. In the combined sample, this association was enhanced after adjusting for the PCOS status (before: $\beta=-.38, P=1.77 \times 10^{-13}$; after: $\beta=-.42$, $P=3.33 \times 10^{-16}$ ), suggesting the genetic effect is independent of the PCOS status. The rs2300441 explained sevenfold higher proportion of the FSH variance than the total variance explained by the two previously reported FSHR missense variants (rs2300441 $R^{2}=1.40 \%$ vs rs6166 $R^{2}=0.17 \%$, rs6165 $R^{2}=0.03 \%$ ). GWAS for LH did not identify any genome-wide significant associations. In conclusion, we identified genome-wide significant association between variants in FSHR and circulating FSH first, with the top associated variant rs 2300441 might be a primary contributor at the population level.
\end{abstract}

KEYWORDS

FSH, FSHR, genetic association, GWAS, LH 


\section{1 | INTRODUCTION}

Follicle-stimulating hormone (FSH) and luteinizing hormone (LH) are pituitary-secreted gonadotropins that are essential for puberty and fertility in both females and males. Abnormal high FSH level during the reproductive years is a diagnostic parameter for premature ovarian failure/aging, while diminished secretion of FSH in combination with increased LH levels can result in failure of gonadal function manifesting polycystic ovary syndrome (PCOS), the most common endocrine disorder among reproductive age women. ${ }^{1}$

Twin studies have shown that the heredity of circulating FSH and $\mathrm{LH}$ levels accounted for $50 \%$ or more of the phenotype variance. ${ }^{2,3}$ To date, five genome-wide association studies (GWASs) for FSH and/or $\mathrm{LH}$ have been conducted, ${ }^{4-7}$ which have identified three genomic loci harboring FSHB, CYP19A1, and LHB genes with genome-wide significant effects on the circulating $\mathrm{LH}$ and/or FSH levels. Candidate gene studies have found DNA variants within or close to $A M H, A M H R 2$, CYP19A1, FSHR, FSHB, LHB, MTHFR, SLC18A2, THADA, and VDR genes showing nominally significant association with FSH and $\mathrm{LH}$ levels. ${ }^{8-25}$ However, these reported variants together could only explain a small proportion $(<3 \%)$ of the phenotype variance, ${ }^{4}$ suggesting that the genetic basis of FSH and $\mathrm{LH}$ is yet poorly understood.

In this study, we conducted the first GWAS for FSH and $\mathrm{LH}$ in Chinese women of Han origin, including 1882 PCOS patients and 708 female controls, ${ }^{26,27}$ which represent a unique resource for comparing the genetic effects on FSH/LH in women with and without PCOS.

\section{2 | METHODS}

\subsection{Study population}

All participants are of Chinese Han origin, including 731 PCOS patients and 890 female controls individuals as described in our previous study, ${ }^{26}$ and additional 1498 PCOS patients described in another previous study. ${ }^{27}$ PCOS was diagnosed according to the Revised 2003 Consensus on Diagnostic Criteria and Long-term Health Risks Related to PCOS. ${ }^{28}$ Any two of the following three criteria were required to be met: oligo- and/or anovulation, clinical and/or biochemical signs of hyperandrogenism, and ovarian morphology showing characteristic polycystic features on ultrasound. Other causes of oligomenorrhea or hyperandrogenism (eg, nonclassical 21-hydroxylase deficiency, Cushing's syndrome, hyperthyroidism, significant elevations in serum prolactin) were excluded on clinical grounds. Controls were gathered primarily from healthy women who presented with regular menstrual cycles, excluding hyperandrogenism and PCOS. All individuals who were taking medications such as oral contraceptives during last 3 months were excluded. Hormone level was drawn on days 2-4 after menses. FSH and LH levels were measured by a chemiluminescent analyzer (Beckman Access Health Company, Chaska, Minnesota). The study was approved by Institutional Review Board of Reproductive Medicine Center of Shandong University and written informed consents were obtained from all subjects.

\subsection{DNA isolation, genotyping, and imputation}

Genomic DNA was extracted from ethylene diamine tetraacetic acidanticoagulated venous blood samples using Flexi Gene DNA kits (Qiagen). PCOS patients and control individuals were genotyped using the Affymetrix Genome-Wide Human SNP Array 6.0 or Axiom Genome-Wide Arrays. Genotype imputation was conducted using IMPUTE version $2^{29,30}$ using the public 1000 Genomes Project Phase 3 imputation panel. ${ }^{31}$ This panel included 2504 worldwide subjects, including 504 from East Asians. Imputed SNPs with imputation information $<80 \%, \mathrm{MAF}<1 \%$, missing rate $>3 \%$ and $\mathrm{HWE}<1 \times 10^{-4}$ were removed from further analysis. Finally, 3101376 genotyped and imputed SNPs passed the quality control.

\section{3 | GWAS analysis}

A series of GWASs were conducted separately for FSH and $\mathrm{LH}$ and separately in the PCOS patients, the controls, and in all individuals, with or without adjusting for the PCOS status. A genomic principal components analysis (PCA) conducted using pca function in PLINK V1.9. ${ }^{32}$ We used unsupervised clustering analysis to cluster the top main PCAs into three clusters. The GWAS was conducted using linear regression assuming an additive allele effect and was adjusted for age, body mass index (BMI), and the top three PCAs using the linear function in PLINK V1.9. The distributions of the observed $P$ values from the GWASs were inspected against the null, that is, the uniform distribution between 0 and 1 using Q-Q plots. ${ }^{33}$ The inflation factor ${ }^{34}$ was close to $1.0(\lambda<1.004)$ in all GWAS and thus not further considered. GWAS results were visualized using Manhattan plots. The $P$-values equal to or smaller than $5 \mathrm{e}-8$ were considered as genome-wide significant and equal to or smaller than $1 \mathrm{e}-5$ were considered as genomewide suggestive evidence of association. Regional linkage disequilibrium (LD) analysis was conducted using the study sample and regional association plots were produced using LocusZoom. ${ }^{35}$ Conditional analysis was carried out for the associated loci by adjusting the genotype of the top-associated SNPs. Explained phenotypic variance was derived for associated SNPs using backward stepwise linear regression analyses.

\subsection{Function annotation}

Potential functions of our GWAS identified SNPs were investigated using public data downloaded from UCSC, including ChIP-seq of transcription factors and Chromatin state discovery and characterization (ChromHMM). ChromHMM is an automated computational approach to segment and annotate the genome, using histone modifications data from nine cell lines. The target gene of enhancer regions was predicted by Dragon ENhancers database (DENdb) which defined the nearest gene of each enhancer by integrating chromatin interaction data.

The Genotype-Tissue Expression project (GTEx) ${ }^{36}$ data set was used to annotate our GWAS SNPs for significant cis-eQTL effects 
and expression levels of the identified genes in all available tissues. The nominal $P$-value of each variant-gene pair was calculated from the genome-wide empirical $P$-value and the beta distribution model of each gene. The variant-gene pairs with a $P$-value lower than the gene-level threshold ( 0.05 false discovery rate [FDR]) was considered significant to be included in the list of variant-gene pairs.

\section{5 | Integration with previous association studies}

To integrate our findings with the main findings from the previous FSH and LH GWAS and candidate gene studies, we looked up the association signals for 11 SNPs reported by previously candidate gene studies $^{8-24}$ and four SNPs reported by previous GWAS. ${ }^{4-7,37}$ Genotypes of 10 SNPs in AMHR2, CYP19A1, FSHR, FSHB, SLC18A2, $T H A D A$, and VDR genes were available in our samples. Multiple linear regressions were performed to address the independent effects of the 11 candidate SNPs, while $P$-values were adjusted for multiple testing using Bonferroni correction of six LD regions. Adjusted $P$-value smaller than or equal to .05 were considered as statistically significant. All statistical analyses were conducted in $R$ (version 3.4.1) unless otherwise specified.

\section{3 | RESULTS}

\section{1 | Sample characteristics}

This study included 2229 PCOS patients and 890 female controls of Chinese Han origin. All controls had regular menstrual cycles, without hyperandrogenism and were on average 2.39 years older than patients $(P<.001)$. As expected, the PCOS patients had a significantly increased BMI $(P<.001)$, LH level $(P<.001)$, and testosterone level $(P<.001))$, as well as a significantly decreased FSH level $(P<.001)$ than controls (Table 1).

TAB LE 1 Characteristics of 3119 female Chinese Han participants

\begin{tabular}{|c|c|c|c|c|}
\hline \multirow[b]{2}{*}{ Characteristics } & \multicolumn{2}{|c|}{ Controls $(\mathrm{N}=890)$} & \multicolumn{2}{|c|}{ PCOS patients $(\mathrm{N}=2229)$} \\
\hline & Mean & SD & Mean & SD \\
\hline Age (years)* & 30.69 & 4.62 & 28.3 & 3.62 \\
\hline BMI* $^{*}$ & 22.68 & 3.12 & 24.75 & 4.01 \\
\hline $\mathrm{FSH}(\mathrm{IU} / \mathrm{L})^{*}$ & 7.54 & 1.97 & 6.63 & 1.68 \\
\hline LH (IU/L)* & 4.75 & 2.08 & 11.61 & 5.49 \\
\hline $\mathrm{T}\left(\mathrm{ng} \mathrm{dL} \mathrm{L}^{-1}\right)^{*}$ & 36.49 & 14.02 & 81.95 & 18.5 \\
\hline
\end{tabular}

Note: Age of subjects with PCOS was that at diagnosis.

Abbreviations: BMI, body mass index; $\mathrm{FSH}$, follicle-stimulating hormone; $\mathrm{LH}$, luteinizing hormone; PCOS, polycystic ovarian syndrome; T, testosterone.

${ }^{*} P<.001$.

\section{2 $\quad$ Genome-wide association studies}

Unsupervised clustering of genomic PCAs showed that our samples were clustered together with East Asians and clearly separated from Europeans and Southern Asians (Figure S1). A set of GWASs for circulating FSH level was separately conducted in 1882 PCOS patients, in 708 controls, and in 2590 combined samples. These GWASs identified a total of 88 SNPs on 17 loci showing genome-wide suggestive association $(P \leq 1 \mathrm{e}-5)$, among which 14 SNPs in a single genomic region spanning $\sim 331.8 \mathrm{~kb}$ on chromosome $2 \mathrm{p} 16.3$ showing genomewide significant association $(P \leq 5 \mathrm{e}-8)$ with the FSH level (Figure $1 \mathrm{~A}$, $B$, Figure S2, Table 2, and Table S1). This region harbored five known genes, including STON1, STON1-GTF2A1L, GTF2A1L, LHCGR, and FSHR (Figure 1C). The top associated SNP rs2300441 was located in the intron 8 or 9 of FSHR, and the A allele was associated with a decreased circulating FSH level. This SNP showed genome-wide significant association with FSH in PCOS patients $\left(P=6.70 \times 10^{-14}\right.$, $\beta=-.43 \mathrm{IU} / \mathrm{L}$ with $95 \% \mathrm{Cl}=-0.31$ to -0.55$)$ and nominally significant association in controls $\left(P=6.52 \times 10^{-4}, \beta=-.35 \mathrm{IU} / \mathrm{L}\right.$ with $95 \%$ $\mathrm{Cl}=-0.15$ to -0.55 , Table 2). The allele effect in the controls and PCOS patients are similar, which indicates the association between rs2300441 and FSH level is independent of PCOS status.

The GWASs in all individuals, with or without adjusting for the PCOS status, again found the 2p16.3 as the only genome-wide significant locus and the rs2300441 being the top-associated SNP. Interestingly, the association signal became more significant after adjusting for the PCOS status (unadjusted $P=1.77 \times 10^{-13}, \beta=-.38 \mathrm{IU} / \mathrm{L}$ with $95 \% \mathrm{Cl}=-0.28$ to -0.48 ; adjusted $P=3.33 \times 10^{-16}, \beta=-.42 \mathrm{IU} / \mathrm{L}$ with $95 \% \mathrm{Cl}=-0.32--0.52$; Table 2 and Figure S2). These results strongly suggest that the effect of rs2300441 on FSH levels is independent of the PCOS status.

GWASs for LH in the PCOS patients, the controls, and all individuals did not reveal any genome-wide significant associations. The most significant signal is rs185780876 $\left(P=3.88 \times 10^{-7}\right)$ in 720 controls.

\section{3 | Conditional analysis and integration with previous literature}

Two missense variants in the exon 10 of FSHR have been reported to be associated with FSH levels in previous candidate gene studies but not in GWAS. ${ }^{8-11}$ These two missense variants also showed genomewide significant association with FSH levels in our study (rs6166 $P=7.52 \times 10^{-10}$ and $\mathrm{rs} 6165 P=2.67 \times 10^{-9}$ ). Conditioning on the genotype of the top-associated SNP rs2300441, these two missense variants showed nominally significant residual effects on FSH levels (rs6165 $P=.01$; rs6166 $P=.002$ Figure 1D, Table S2). Further conditioning on the genotype of both rs2300441 and rs6166, no nominally significant signals were observed in this region ( $P>.05$, Table S2). A moderate LD between rs2300441 and the two missense variants was observed $\left(r^{2}=0.25\right)$ while the LD between rs6165 and rs6166 was very high $\left(r^{2}=0.88\right)$. A backward stepwise regression was conducted to partition the independent contributions of these three variants. 


\section{(A)}

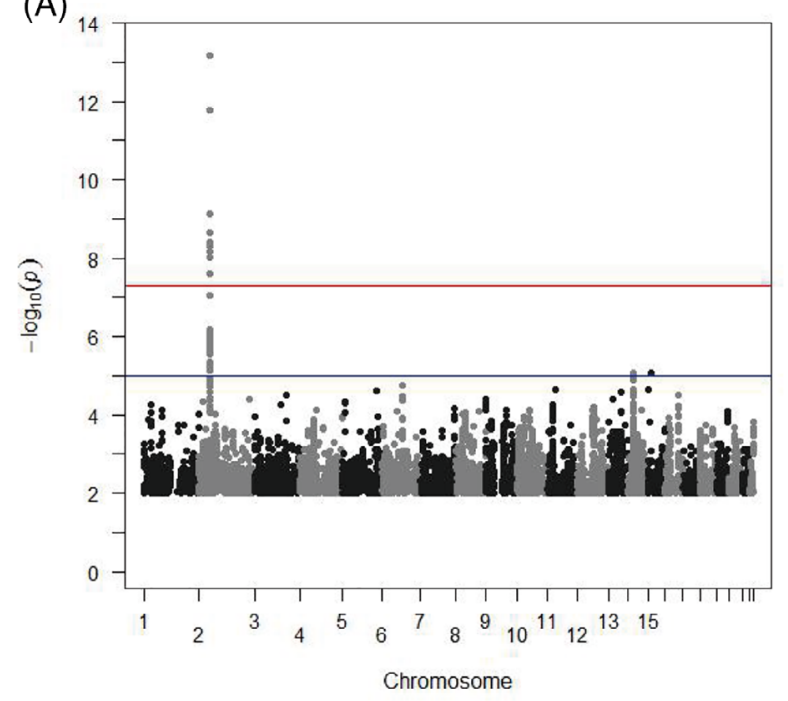

(C)

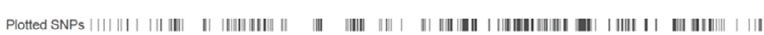

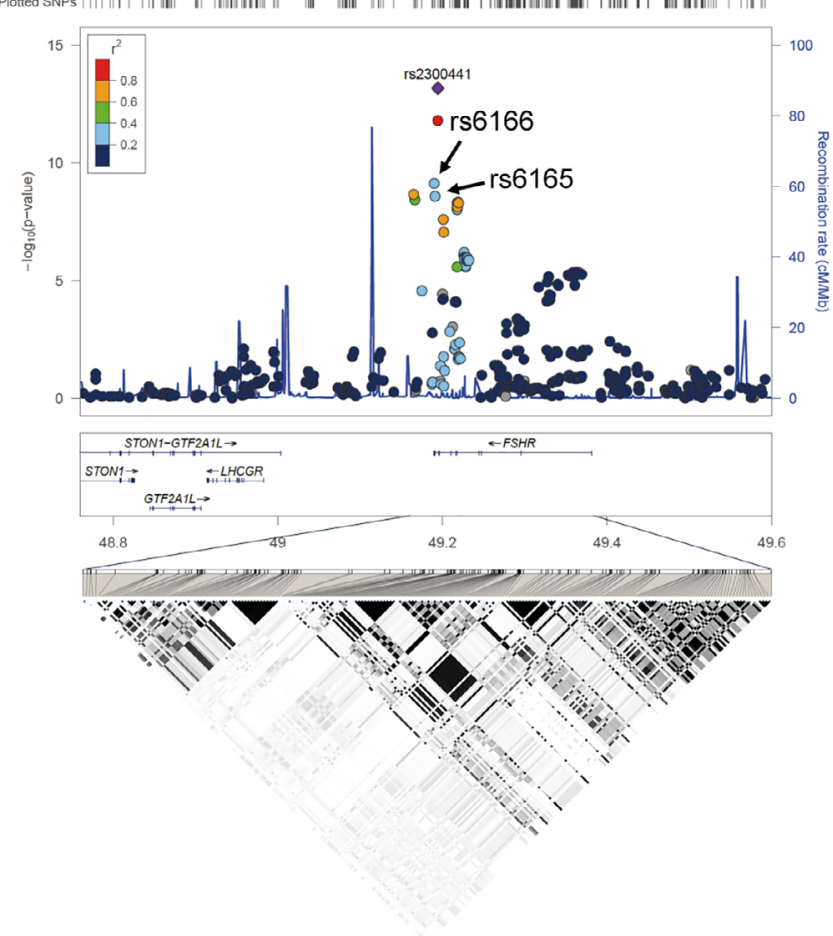

(B)

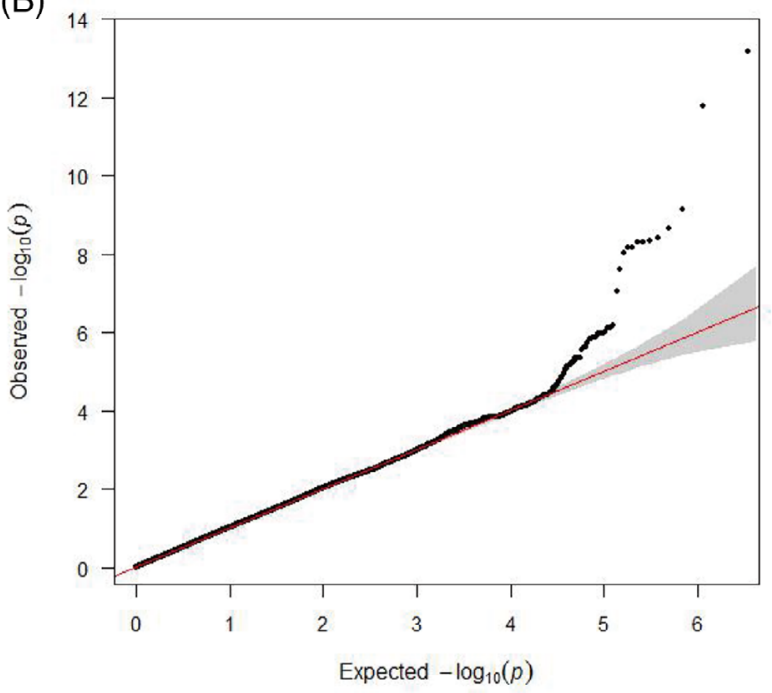

(D)

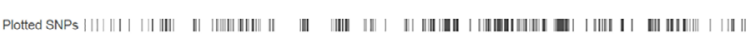

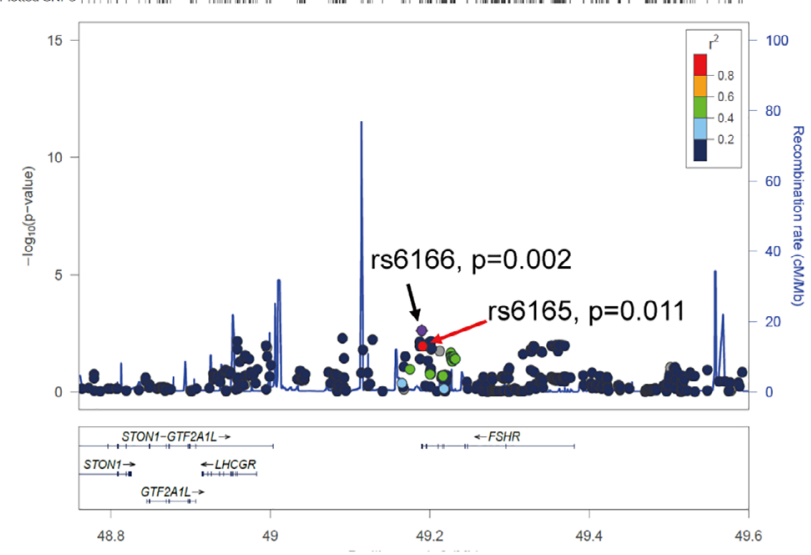

FIGURE 1 GWAS analysis for FSH level in 1882 PCOS patients with age and BMI as covariates. A, Manhattan plot for SNPs associated on FSH level. B, Quantile-quantile plot showing the distribution of expected compared to observed -log10 P for the association test results. C, Regional Manhattan plots. Chromosome 2p16.3 (48.76-49.60 Mb) containing FSHR. The -log10 P values of all SNPs are plotted against their physical positions (hg19). Known genes (blue lines) are aligned along their genomic position. At the bottom, the LD structure is shown as obtained from study samples. Light colors indicate low LD; darker colors indicate high LD. D, Regional Manhattan plots for FSH level conditioned on rs2300441 (with rs2300441, age, and BMI as covariates). FSH, Follicle-stimulating hormone; GWAS, genome-wide association study; LD, linkage disequilibrium; PCOS, polycystic ovarian syndrome [Colour figure can be viewed at wileyonlinelibrary.com]

The rs2300441 explained the largest proportion of the FSH variance (rs2300441 $R^{2}=1.40 \%$ ), which was sevenfold larger than the combined variance attributable to the two missense variants (rs6166 $R^{2}=0.17 \%$, rs6165 $R^{2}=0.03 \%$ ). These results overall suggest that rs2300441 and the two missense variants may influence FSH independently, while the primary contribution was from rs2300441.

\subsection{Functional annotation}

Potential functions of our GWAS identified SNPs were investigated using multiple types of public data. ChromHMM revealed that four of our candidate SNPs (rs2072484, rs2072483, rs13032037, and rs12620721) are contained within enhancers characterized from 


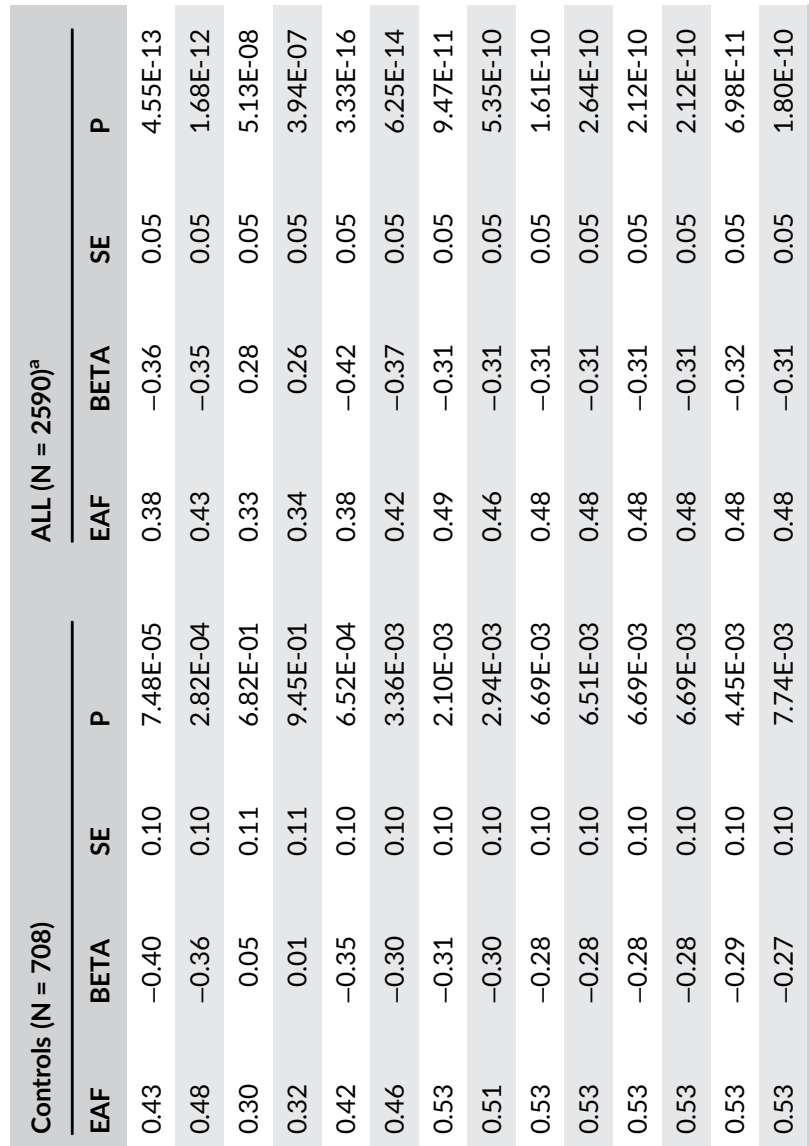

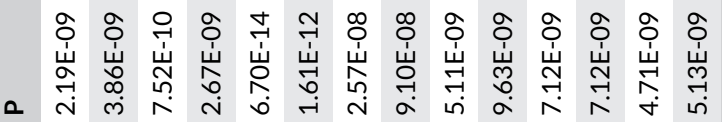

ய

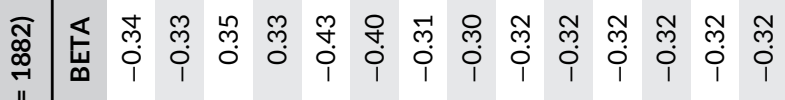

בั

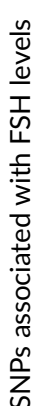

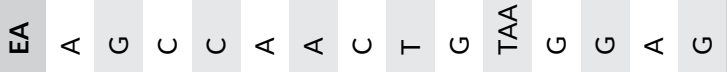

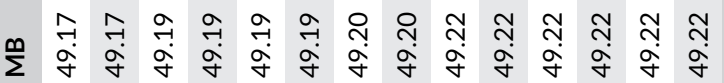

选

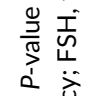




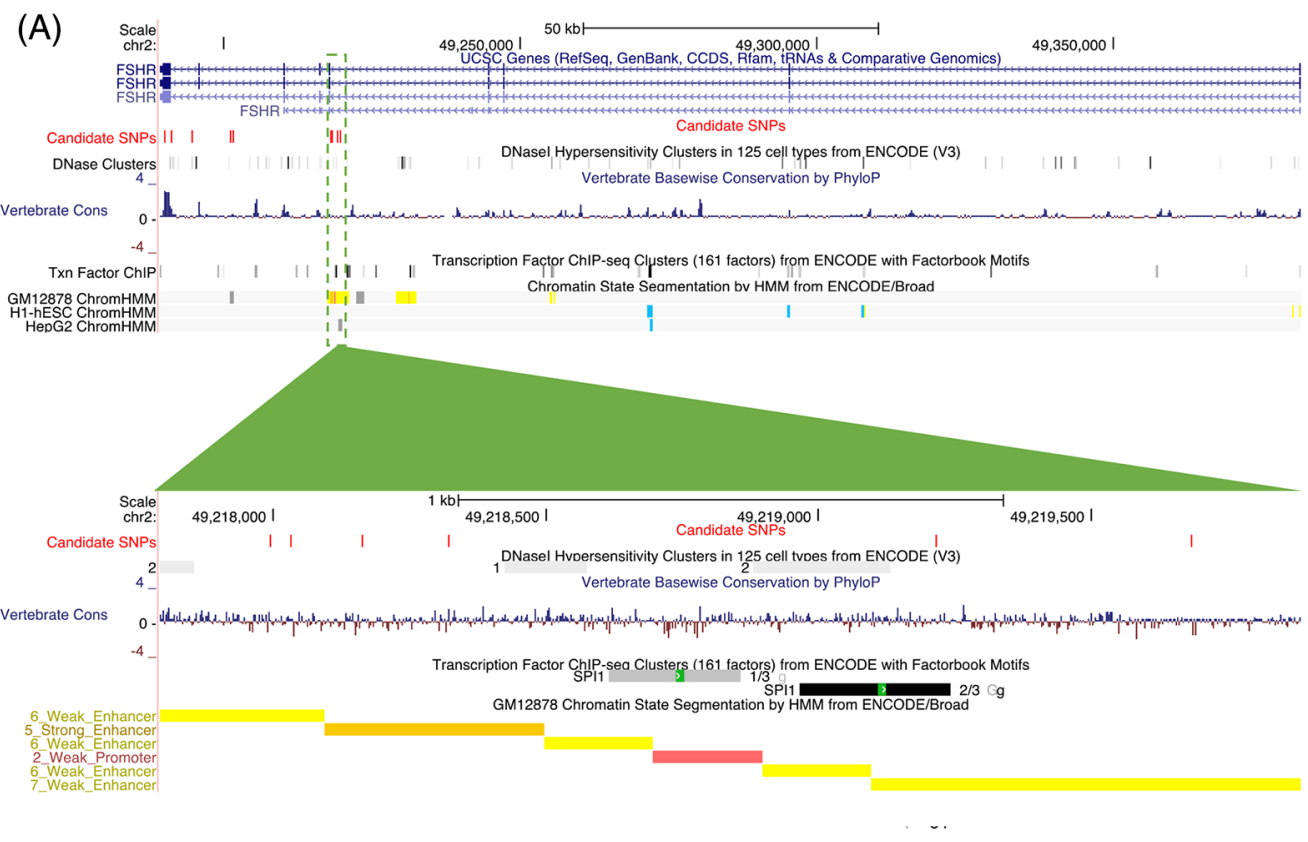

(B)

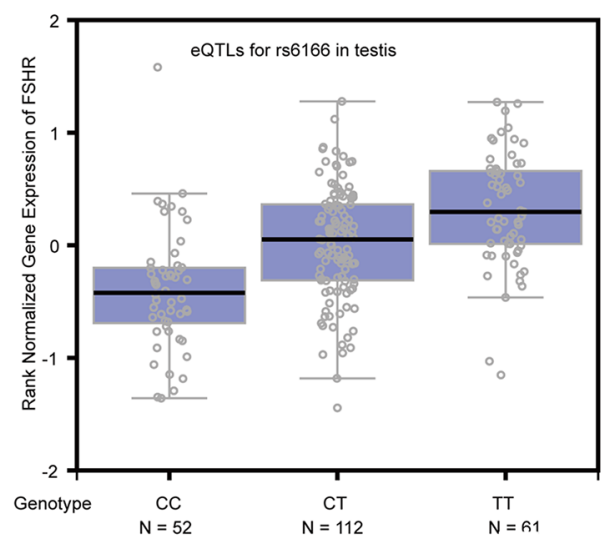

(D)

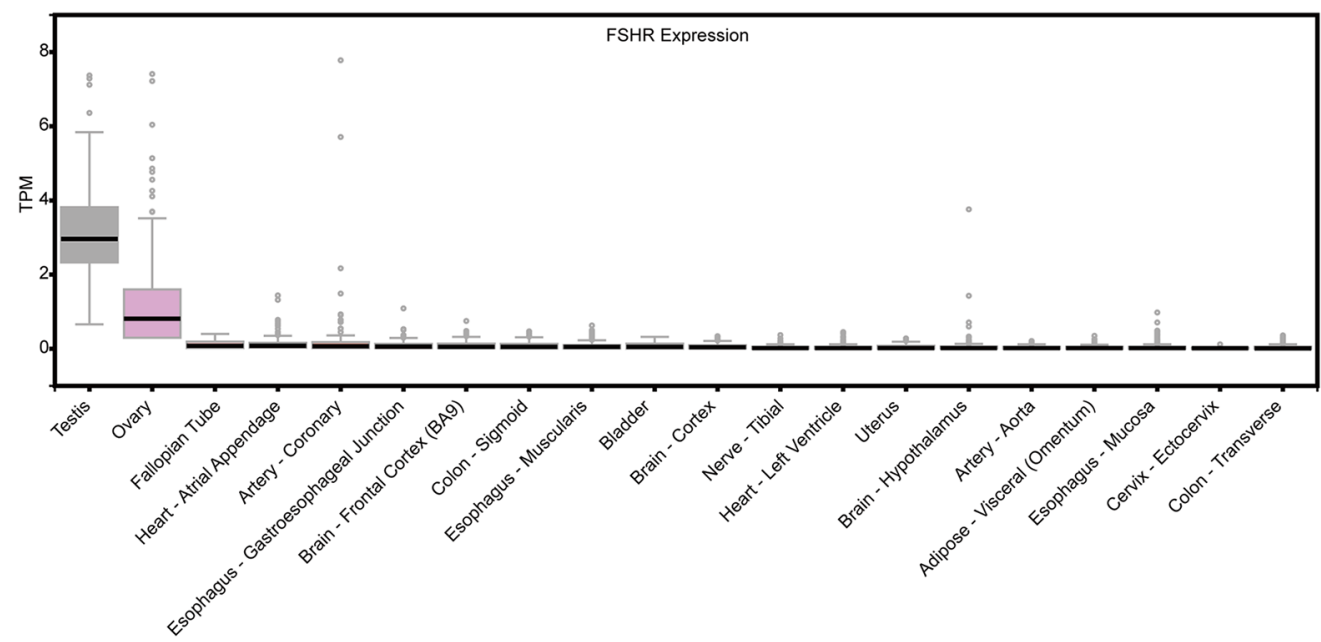

FIGURE 2 Functional annotation of candidate SNPs. A, The upper half part: A UCSC Genome Browser global view of FSHR gene. In order, the features indicate (1) RefSeq gene, (2) the locations of 14 candidate SNPs, (3) DNase I hypersensitivity clusters, (4) conservation scores, (5) transcription factor ChIP-seq, and (6) chromatin state segmentation in three cell lines. The green box represents a region located inside one enhancer in GM12878, including four SNPs (rs2072484, rs2072483, rs13032037, and rs12620721). The lower half part: Genomic features of four candidate SNPs (rs2072484, rs2072483, rs13032037, and rs12620721).

From top to bottom, the features shown are (1) RefSeq gene, (2) the locations of four candidate SNPs, (3) DNase I hypersensitivity clusters, (4) conservation scores, (5) transcription factor ChIP-seq, and (6) chromatin state segmentation in GM12878 (orange and yellow bars are weak and strong enhancers, respectively). B,C, FSHR is a genome-wide significant eQTL for rs6166 and rs2300441 in testis. D, FSHR specifically express in ovary and testis. Expression values are shown in transcripts per million (TPM), calculated from a gene model with isoforms collapsed to a single gene. No other normalization steps have been applied. Box plots are shown as median and 25th and 75th percentiles; points are displayed as outliers if they are above or below 1.5 times the interquartile range [Colour figure can be viewed at wileyonlinelibrary.com] patterns of histone modifications in GM12878 cell line. This region is enriched with binding peaks of one transcription factor SPI1. Furthermore, this region was linked to its target gene FSHR by chromatin interaction assays (DENdb), suggesting a self-regulated pattern. These findings strongly support a functional role of the 14 GWAS-identified SNPs (Figure 2A).

The GTEx ${ }^{36}$ data set was used to annotate our GWAS SNPs for significant cis-eQTL effects in all available tissues. Among the 14 
identified variants, 11 showed significant association with the expression of FSHR in testis (Table S3) and the most significant SNPs were rs6166 $(P=9.20 \mathrm{E}-13)$ and rs2300441 $\left(P=1.00 \times 10^{-11}\right)$. Interestingly, the FSH-reducing A allele of rs2300441 and T allele of rs6166 was associated with an increased expression of FSHR ( $r$ 2300441 effect size $=0.41$, rs6166 effect size $=0.43$; Table S3 and Figure 2B, C). Moreover, based on GTEx gene expression data, FSHR was expressed specifically in testis and ovary (Figure 2D). These results indicated a direct functional link between rs2300441 and FSH action in gonads although the underlying mechanism needs further study.

\section{5 | Candidate gene replication}

To integrate our results with the main findings of previous reports, we looked up the association signals for 10 SNPs in AMHR2, CYP19A1, FSHR, FSHB, SLC18A2, THADA, and VDR genes from the previous $\mathrm{LH}$ and FSH GWAS (Table S4) and candidate gene studies (Table S5). Previous GWASs have identified CYP19A1 and FSHB gene variants being involved in FSH levels, ${ }^{4,6,37}$ and $F S H B$ and $L H B$ variants involved in $\mathrm{LH}$ levels. ${ }^{4,5,7}$ In our study, the FSHB variants (rs10835638, rs11031005) also showed nominally significant effects on FSH level in controls, and $\mathrm{LH}$ level in controls and in the combined samples ( $P$-adjusted $<.05$, Table S6) while the SNP at CYP19A1 was not replicated. Previous candidate gene studies have suggested a link between LH and FSH levels and the two missense FSHR variants, ${ }^{8-11}$ which showed genome-wide significant association with FSH levels in our GWAS, also showed nominally significant association with LH levels in the combined samples (rs6165 and rs6166, P-adjusted <.05, Table S6). The effects of previously reported gene variants in or close to THADA, SLC18A2, and VDR were not replicated in our sample $(P>.05)$. Thus, besides the delivery of a novel variant at FSHR showing genome-wide significant association with FSH, we confirmed the effect of the variants at FSHR and FSHB gene variants on both FSH and LH levels as suggested in previous candidate gene studies.

\section{6 | Discussion}

In our study, the 2p16.3 FSHR region showed genome-wide significant association with FSH level in Han Chinese women. This locus has also been reported to be associated with multiple sex hormone related phenotypes in previous GWAS studies. For example, our previous GWAS, which contained the samples of the current study identified two different SNPs at the FSHR gene region showing significant association with PCOS risk. ${ }^{27}$ A previous GWAS in 22000 Europeans identified several different SNPs in the same region showing suggestive association with sex hormone-binding globulin concentrations. ${ }^{38}$ This locus has also been suggested as a susceptibility locus for erectile dysfunction, although was not replicated by Bovijn et al. ${ }^{39,40}$ Therefore, multiple lines of evidence in population samples, together with our findings, underlie the important role of FSHR in sex hormone genesis. The failure of detecting the FSHR association with FSH levels in previous $\mathrm{GWAS}^{4,6,37}$ could be due to several factors, such as sample size, sample demographic characteristics, and population heterogeneity.

Two missense variants in the exon 10 of FSHR ( $r$ 6165, Thr307Ala, and rs6166, Asn680Ser) have been associated with FSH levels in previous candidate gene studies. ${ }^{8-11}$ We also replicated their effects with genome-wide significant evidence in our GWAS. These two variants in the key domain of FSHR play important roles in FSH efficacy. ${ }^{41}$ In our study, the top-associated SNP rs2300441 is located in the intron 8 or 9 of FSHR. Our data highlight that the effect of rs2300441 was independent of rs6165 and rs6166. In addition, the effect of rs2300441 was much stronger than those of rs6165 and rs6166 in a multivariable model. It is possible that rs2300441 has effects on FSHR expression or action, resulting in a need for altering FSH levels to stimulate the receptor for follicle growth.

In GTEx, FSHR was an eQTL of rs2300441 meeting genome-wide significance in testis but is not significant in ovary. Since FSHR is specifically expressed in ovarian granulosa cells, further experiments in granulosa cells are needed to confirm the association of rs2300441 and the expression of FSHR. ${ }^{42}$

FSHR SNPs have been correlated with ovarian reserve and response. Mutations and variants of FSHR were considered as a risk factor for ovarian hyperstimulation syndrome occurrence in assisted reproduction techniques (ARTs). ${ }^{43}$ FSH and FSHR might affect controlled ovarian stimulation in ART and consequently assisted reproduction outcome. ${ }^{43,44}$ Combination of FSHR genotype and other risk factors may provide a mean of predicting ovarian response to $\mathrm{FSH}$ or chance of pregnancy before in vitro fertilization procedures are initiated. ${ }^{45,46}$ Variant rs2300441 identified in this study could be a potential marker for ovarian response and reproduction outcome in ARTs.

\section{CONCLUSIONS}

In conclusion, the variant rs2300441 in FSHR showed a genome-wide significant effect on FSH levels in both PCOS patients and female controls of Chinese Han origin. The effect of rs2300441 on FSH levels was independent of PCOS status and the two previously reported FSHR missense variants.

\section{ACKNOWLEDGEMENTS}

This study was funded by the National Key Research and Development Program of China (2017YFC1001000), the National Natural Science Foundation of China (31571548, 81622021, 81701410, and 31601199), the National Natural Science Foundation of Shandong Province (JQ201816). The authors especially thank all the participants in this study.

\section{AUTHORS' CONTRIBUTION}

Jinting Yan, Ye Tian, Fan Liu, and Han Zhao designed research, analyzed and interpreted data; Xingjian Gao, Yongzhi Cao, and Fuduan Peng assisted data analysis; Ye Tian, Linlin Cui, Yunna Ning, Yongzhi Cao, and Li You contributed experimental regents and performed 
experiments; Jinting Yan, Ye Tian, and Fan Liu mainly wrote the manuscript; Han Zhao provided critical comments to the manuscript, Fan Liu and Han Zhao supervised this work. All authors read and approved the final version of the manuscript.

\section{CONFLICT OF INTEREST}

The authors declare no potential conflict of interest.

\section{DATA AVAILABILITY STATEMENT}

The data that support the findings of this study are available from the corresponding author upon reasonable request.

\section{ORCID}

Jinting Yan (D) https://orcid.org/0000-0002-6499-2681

\section{REFERENCES}

1. Taylor AE, McCourt B, Martin KA, et al. Determinants of abnormal gonadotropin secretion in clinically defined women with polycystic ovary syndrome. J Clin Endocrinol Metab. 1997;82(7):2248-2256.

2. Meikle AW, Stephenson RA, Lewis CM, Wiebke GA, Middleton RG. Age, genetic, and nongenetic factors influencing variation in serum sex steroids and zonal volumes of the prostate and benign prostatic hyperplasia in twins. Prostate. 1997;33(2):105-111.

3. Kuijper EA, Lambalk CB, Boomsma DI, et al. Heritability of reproductive hormones in adult male twins. Hum Reprod. 2007;22(8):21532159.

4. Ruth KS, Campbell PJ, Chew S, et al. Genome-wide association study with 1000 genomes imputation identifies signals for nine sex hormone-related phenotypes. Eur J Hum Genet. 2016;24(2):284-290.

5. Hayes MG, Urbanek M, Ehrmann DA, et al. Genome-wide association of polycystic ovary syndrome implicates alterations in gonadotropin secretion in European ancestry populations. Nat Commun. 2015;6: 7502 .

6. Chen Z, Tao S, Gao Y, et al. Genome-wide association study of sex hormones, gonadotropins and sex hormone-binding protein in Chinese men. J Med Genet. 2013;50(12):794-801.

7. Sun BB, Maranville JC, Peters JE, et al. Genomic atlas of the human plasma proteome. Nature. 2018;558(7708):73-79.

8. Busch AS, Hagen CP, Almstrup K, Main KM, Juul A. Genetic variations altering FSH action affect circulating hormone levels as well as follicle growth in healthy peripubertal girls. Hum Reprod. 2016;31(4): 897-904.

9. Trevisan CM, Peluso C, Cordts EB, et al. Ala307Thr and Asn680Ser polymorphisms of FSHR gene in human reproduction outcomes. Cell Physiol Biochem. 2014;34(5):1527-1535.

10. Perez Mayorga M, Gromoll J, Behre HM, Gassner C, Nieschlag E, Simoni M. Ovarian response to follicle-stimulating hormone (FSH) stimulation depends on the FSH receptor genotype. J Clin Endocrinol Metab. 2000;85(9):3365-3369.

11. Yan $Y$, Gong Z, Zhang L, et al. Association of follicle-stimulating hormone receptor polymorphisms with ovarian response in Chinese women: a prospective clinical study. PLoS One. 2013;8(10):e78138.

12. Grigorova M, Punab M, Punab AM, et al. Reproductive physiology in young men is cumulatively affected by $\mathrm{FSH}$-action modulating genetic variants: FSHR -29G/A and c.2039 A/G, FSHB -211G/T. PLoS One. 2014;9(4):e94244.

13. Henriksen LS, Hagen CP, Assens M, et al. Genetic variations in FSH action affect sex hormone levels and breast tissue size in infant girls: a pilot study. J Clin Endocrinol Metab. 2016;101(8):3191-3198.

14. Achrekar SK, Modi DN, Meherji PK, Patel ZM, Mahale SD. Follicle stimulating hormone receptor gene variants in women with primary and secondary amenorrhea. J Assist Reprod Genet. 2010;27(6): 317-326.

15. La Marca A, Papaleo E, Alviggi $C$, et al. The combination of genetic variants of the FSHB and FSHR genes affects serum FSH in women of reproductive age. Hum Reprod. 2013;28(5):1369-1374.

16. Hagen CP, Aksglaede L, Sorensen K, et al. FSHB-211 and FSHR 2039 are associated with serum levels of follicle-stimulating hormone and antimullerian hormone in healthy girls: a longitudinal cohort study. Fertil Steril. 2013;100(4):1089-1095.

17. Busch AS, Tuttelmann F, Zitzmann M, Kliesch S, Gromoll J. The FSHB $-211 \mathrm{G}>\mathrm{T}$ variant attenuates serum $\mathrm{FSH}$ levels in the supraphysiological gonadotropin setting of Klinefelter syndrome. Eur J Hum Genet. 2015;23(5):700-703.

18. Grigorova M, Punab M, Ausmees K, Laan M. FSHB promoter polymorphism within evolutionary conserved element is associated with serum FSH level in men. Hum Reprod. 2008;23(9):2160-2166.

19. Grigorova M, Punab M, Poolamets $\mathrm{O}$, et al. Increased prevalance of the $-211 \mathrm{~T}$ allele of follicle stimulating hormone (FSH) beta subunit promoter polymorphism and lower serum FSH in infertile men. $J$ Clin Endocrinol Metab. 2010;95(1):100-108.

20. Georgopoulos NA, Karagiannidou E, Koika V, et al. Increased frequency of the anti-mullerian-inhibiting hormone receptor 2 (AMHR2) $482 \mathrm{~A}>\mathrm{G}$ polymorphism in women with polycystic ovary syndrome: relationship to luteinizing hormone levels. J Clin Endocrinol Metab. 2013;98(11):E1866-E1870.

21. Li Q, Yan Z, Kuang Y, et al. Genetic variations in the $3^{\prime}$-untranslated region of SLC18A2 are associated with serum FSH concentration in polycystic ovary syndrome patients and regulate gene expression in vitro. Hum Reprod. 2016;31(9):2150-2157.

22. Sery O, Sramkova T, Klempova J, Stastny F, Lochman J, Khan NA. The relationship between the C677T polymorphism of the MTHFR gene and serum levels of luteinizing hormone in males with erectile dysfunction. Neuro Endocrinol Lett. 2012;33(5):499-504.

23. Cui L, Zhao H, Zhang B, et al. Genotype-phenotype correlations of PCOS susceptibility SNPs identified by GWAS in a large cohort of Han Chinese women. Hum Reprod. 2013;28(2):538-544.

24. Ranjzad F, Mahban A, Shemirani Al, et al. Influence of gene variants related to calcium homeostasis on biochemical parameters of women with polycystic ovary syndrome. J Assist Reprod Genet. 2011;28(3): 225-232.

25. Xu P, Shen SM, Zhang XL, et al. Haplotype analysis of single nucleotide polymorphisms in anti-mullerian hormone gene in Chinese PCOS women. Arch Gynecol Obstet. 2013;288(1):125-130.

26. Chen ZJ, Zhao H, He L, et al. Genome-wide association study identifies susceptibility loci for polycystic ovary syndrome on chromosome 2p16.3, 2p21 and 9q33.3. Nat Genet. 2011;43(1):55-59.

27. Shi $\mathrm{Y}$, Zhao H, Shi Y, et al. Genome-wide association study identifies eight new risk loci for polycystic ovary syndrome. Nat Genet. 2012;44 (9):1020-1025

28. Rotterdam EA-SPCWG. Revised 2003 consensus on diagnostic criteria and long-term health risks related to polycystic ovary syndrome. Fertil Steril. 2004;81(1):19-25.

29. Howie B, Marchini J, Stephens M. Genotype imputation with thousands of genomes. G3 (Bethesda). 2011;1(6):457-470.

30. Howie BN, Donnelly P, Marchini J. A flexible and accurate genotype imputation method for the next generation of genome-wide association studies. PLoS Genet. 2009;5(6):e1000529.

31. 1000 Genomes Project Consortium, Auton A, Brooks LD, Durbin RM, et al. A global reference for human genetic variation. Nature. 2015; 526(7571):68-74.

32. Purcell S, Neale B, Todd-Brown K, et al. PLINK: a tool set for wholegenome association and population-based linkage analyses. Am J Hum Genet. 2007;81(3):559-575.

33. Pearson TA, Manolio TA. How to interpret a genome-wide association study. JAMA. 2008;299(11):1335-1344. 
34. Devlin B, Roeder K. Genomic control for association studies. Biometrics. 1999;55(4):997-1004.

35. Pruim RJ, Welch RP, Sanna S, et al. LocusZoom: regional visualization of genome-wide association scan results. Bioinformatics. 2010;26(18): 2336-2337.

36. GTEx Consortium, Laboratory, Data Analysis \&Coordinating Center (LDACC)-Analysis Working Group, Statistical Methods groups-Analysis Working Group; Enhancing GTEx (eGTEx) groups, et al. Genetic effects on gene expression across human tissues. Nature. 2017;550 (7675):204-213.

37. Schuh-Huerta SM, Johnson NA, Rosen MP, Sternfeld B, Cedars MI, Reijo Pera RA. Genetic variants and environmental factors associated with hormonal markers of ovarian reserve in Caucasian and African American women. Hum Reprod. 2012;27(2):594-608.

38. Coviello AD, Haring R, Wellons $M$, et al. A genome-wide association meta-analysis of circulating sex hormone-binding globulin reveals multiple loci implicated in sex steroid hormone regulation. PLoS Genet. 2012;8(7):e1002805.

39. Kerns SL, Ostrer H, Stock R, et al. Genome-wide association study to identify single nucleotide polymorphisms (SNPs) associated with the development of erectile dysfunction in African-American men after radiotherapy for prostate cancer. Int J Radiat Oncol Biol Phys. 2010;78 (5):1292-1300.

40. Bovijn J, Jackson L, Censin J, et al. GWAS identifies risk locus for erectile dysfunction and implicates hypothalamic neurobiology and diabetes in etiology. Am J Hum Genet. 2019;104(1):157-163.

41. Renner M, Goeppert B, Siraj MA, et al. Follicle-stimulating hormone receptor expression in soft tissue sarcomas. Histopathology. 2013;63 (1):29-35
42. Minegishi T, Tano M, Igarashi M, et al. Expression of follicle-stimulating hormone receptor in human ovary. Eur J Clin Invest. 1997;27(6): 469-474.

43. Rizk B. Symposium: update on prediction and management of OHSS. Genetics of ovarian hyperstimulation syndrome. Reprod Biomed Online. 2009;19(1):14-27.

44. Dieterich M, Bolz M, Reimer T, Costagliola S, Gerber B. Two different entities of spontaneous ovarian hyperstimulation in a woman with FSH receptor mutation. Reprod Biomed Online. 2010;20(6):751-758.

45. Riccetti L, De Pascali F, Gilioli L, et al. Genetics of gonadotropins and their receptors as markers of ovarian reserve and response in controlled ovarian stimulation. Best Pract Res Clin Obstet Gynaecol. 2017;44:15-25.

46. Lindgren I, Baath $\mathrm{M}$, Uvebrant $\mathrm{K}$, et al. Combined assessment of polymorphisms in the LHCGR and FSHR genes predict chance of pregnancy after in vitro fertilization. Hum Reprod. 2016;31(3):672-683.

\section{SUPPORTING INFORMATION}

Additional supporting information may be found online in the Supporting Information section at the end of this article.

How to cite this article: Yan J, Tian Y, Gao X, et al. A genomewide association study identifies FSHR rs2300441 associated with follicle-stimulating hormone levels. Clin Genet. 2020;1-9. https://doi.org/10.1111/cge.13741 